\title{
Análise das exposições médicas em mamografia digital
}

\author{
S. R. Oliveira' ${ }^{\text {; N. O. Mantuano }}$; A. S. Albrecht ${ }^{2}$ e L. S. Flor ${ }^{3}$ \\ ${ }^{1}$ Escola Politécnica de Saúde Joaquim Venâncio (EPSJV) \\ Fundação Oswaldo Cruz (FIOCRUZ) \\ Av. Brasil, $n^{\circ} 4365$ - Manguinhos. \\ 21040-360 Rio de Janeiro, RJ. \\ oliveirasr@fiocruz.br \\ 2 Instituto de Física (IF) \\ Universidade Federal do Rio de Janeiro (UFRJ) \\ Av. Athos da Silveira Ramos, n 149 - CT/bloco A - Cidade Universitária. \\ 21941-972 Rio de Janeiro, RJ. \\ nataliamantuano@gmail.com_afonsofismed@gmail.com \\ ${ }^{3}$ Hospital São Vicente de Paulo (HSVP) \\ Rua Dr. Satamini, $n^{\circ} 333$ - Tijuca. \\ 20270-233 Rio de Janeiro - RJ - Brasil \\ leonardo.flor@hsvp.org.br
}

\begin{abstract}
RESUMO
A utilização de equipamentos de mamografia digital no diagnostico precoce de câncer é cada vez maior, principalmente por causa das imagens de alta definição, que possibilitam detectar pequenas alterações. Entretanto, a alta tecnologia associada ao equipamento nos obriga a buscar um aprimoramento por parte das técnicas radiográficas. Com isso, procuramos analisar como a qualificação profissional pode interferir na técnica radiográfica. Para isto, foi realizado levantamento no qual foram avaliados os exames de 1190 pacientes submetidas à rotina de mamografia (entende-se por 4 exposições básicas: 2 crânio caudal e 2 médio lateral oblíquo) no ano de 2013. As exposições médicas analisadas foram obtidas a partir de um único equipamento digital e foram comparados os procedimentos realizados por 3 diferentes profissionais de mamografia. Comparando a variação de técnica radiográfica em relação a profissional que efetuou a exposição, o que se observa é que a profissional B apresentou a melhor conduta em relação à proteção radiológica, pois considerava a densidade da mama na escolha do parâmetro técnico do equipamento. Já as profissionais A, que é recém-formada, e C, que possui mais tempo de serviço, praticamente não realizavam variações no padrão de exposição, mesmo para diferentes densidades mamárias. Desta forma, pode-se concluir que há a necessidade de atualização dos profissionais a fim de que as ferramentas disponíveis de limitação de dose e variabilidade de mamas do mamógrafo digital sejam eficientemente empregadas na rotina do serviço e, consequentemente, atendam os requisitos da legislação vigente.
\end{abstract}

Palavras-chave: Qualificação profissional, Mamografia, Câncer de mama. 


\section{INTRODUÇÃO}

De acordo com os relatórios apresentados pelo Instituto Nacional do Câncer (INCA) nos últimos anos é visível o crescimento do número de casos de mulheres com câncer de mama. Segundo relatos de outras organizações é a principal causa de morte por câncer entre mulheres no mundo todo, entretanto é o tipo que melhor apresenta resultados favoráveis a partir da aplicação de uma política de rastreamento.

Em contra partida, as dificuldades para implantação de um programa desta natureza são inúmeras, que vão desde a falta de profissionais capacitados até unidades de saúde deficitárias para realizar algum tipo de exame ou tratamento. Um programa desta natureza, além de complexo, torna-se muito oneroso e necessita de uma equipe de profissionais técnicos especializados, além de um corpo clínico dedicado.

A implantação de um programa de controle de câncer de mama depende da vontade política por parte do Governo Federal e da organização dos Estados e Municípios em promover ações coletivas visando à atenção a mulher, em especial a população feminina de alto risco para a doença. Hoje, a melhor alternativa para o rastreamento desta população é por meio do exame de mamografia [2].

Por outro lado, é sabido que estas ações ainda não dão conta diretamente do problema principal da saúde brasileira que é a assistência. As unidades básicas de saúde devem estar organizadas para receber e realizar o exame clínico das mamas das mulheres, solicitar exames mamográficos, receber resultados e encaminhar aquelas cujos exames indiquem necessidade de maior investigação [4].

Em estudos realizados em outros estados brasileiros, o que se observa é a falta de estrutura por parte de regiões mais distantes dos grandes centros urbanos ou mesmo nas capitais do país, que em alguns casos não possuem nem uma rede privada que possa atender toda a população de forma igualitária, conforme preconizada nas diretrizes básicas do SUS [5].

Segundo organizações médicas ligadas diretamente a mulher, como o Colégio Brasileiro de Radiologia e Diagnóstico por Imagem (CBR), a Sociedade Brasileira de Mastologia (SBM) e a Federação Brasileira das Associações de Ginecologia e Obstetrícia (FEBRASGO), no Brasil não existe uma política de rastreamento populacional, somente há rastreamento oportunístico. 
Portanto, ações no sentido de padronizar o rastreamento do câncer de mama, assim como esclarecer a população sobre a importância da sua realização, devem ser estimuladas [6].

De acordo com a Lei 11.664, de 2008, sancionada pelo presidente Luis Inácio, à época, e decretada pelo Congresso Nacional, ficou estabelecido, a partir das ações de saúde previstas no caput e inciso II do art. $7^{\circ}$ da Lei 8.080 de 1990, que o Sistema Único de Saúde (SUS) deve assegurar, por meio de seus serviços próprios, conveniados ou contratados a assistência integral à saúde da mulher, incluindo amplo trabalho de comunicação, detecção, tratamento e controle do câncer de mama das pacientes submetidas à rede e obrigando a realização do exame mamográfico a todas as mulheres a partir dos 40 anos de idade [1].

Entretanto, a política atual de atenção ao combate do câncer de mama, divulgado no Programa Nacional de Combate ao Câncer de Mama, editado em 2010 e revisado em 2011, estipula que a prática de rastreamento, com uso da mamografia, deve ser realizada apenas em mulheres na faixa etária de 50 a 69 anos e em periodicidade bienal. Para as mulheres na faixa etária de 40 a 49 anos a recomendação é o exame clínico podendo ser realizado o de mamografia para casos de alteração clínica [3].

Este trabalho faz parte de um projeto maior que busca estudar a fundo a qualidade da aplicação dos exames de Mamografia do Estado do Rio de Janeiro, apurando aspectos técnicos como a qualidade dos equipamentos, os profissionais que realizam o exame e as políticas públicas implantadas em cada uma das regiões do estado para atender aos requisitos descritos na legislação.

Como proposta preliminar de um estudo maior, este trabalho teve por objetivo verificar como é processo de capacitação/formação das profissionais técnicas em radiologia, que realizam, exclusivamente, procedimentos em mamografia, e de que forma isto interfere no processo de trabalho e na aplicação das técnicas durante a realização de um exame.

\section{METODOLOGIA}

O presente trabalho foi realizado no ano de 2013, no período entre janeiro e dezembro, em um Serviço de Radiologia de um hospital privado da cidade do Rio de Janeiro, que possui uma rotina 
de mamografia nos dias úteis. Não foram coletados dados de exames que supostamente pudessem ser realizados no final de semana (período fora de rotina no hospital), nos dias de paradas para manutenção ou correções de problemas diversos e em dias em que a agenda de marcação foi suspensa por problemas outros.

Com isso foram obtidos 1190 exames de mamografia de diferentes pacientes, que foram submetidas à rotina de mamografia, ou seja, apenas mulheres que obtiveram imagens de ambas as mamas nas projeções crânio caudal e médio lateral oblíqua. Foram descartadas imagens complementares e exames de mulheres com próteses.

Por se tratar de um estudo preliminar, a metodologia adotada para este trabalho foi a de estudo de caso, uma vez que foi realizado totalmente em uma única unidade de saúde. Entretanto, como a ideia é relacionar os conhecimentos existentes com a prática realizada, isto não foi um problema, pois os procedimentos realizados no hospital não diferem muito dos realizados nas unidades da saúde pública, além disso o volume de pacientes atendidos também não difere tanto da maioria das unidades públicas da cidade do Rio de Janeiro.

As imagens radiológicas analisadas de cada paciente foram obtidas a partir de um equipamento totalmente digital, modelo Senographe DS. Todo o trabalho foi realizado por três profissionais técnicas em radiologia (A, B e C) apenas, que variavam as técnicas de exposição pré-definidas pelo equipamento de acordo com os tipos de densidade da mama.

Os parâmetros estipulados no equipamento são: contraste (CNT), geralmente aplicado para mamas densas (tipo 4); padrão (STD), recomendado para mamas que apresentem os dois tipos de tecidos (50\% glandular e 50\% adiposo); e técnica de redução de dose (DOSE), indicado para mamas praticamente adiposas (tipo 1).

Sobre a qualificação das profissionais podemos destacar que a profissional A tem pouco tempo de formada e sua prática ocorreu no próprio local de trabalho, além do que não possui título de especialista. Por outro lado, a profissional B é a que possui maior conhecimento entre as três, além de ter o título de especialista. Já a profissional C tem bastante experiência no próprio serviço, com maior tempo de casa e com especialização de longa data. 


\section{RESULTADOS E DISCUSSÃO}

Os dados absolutos, bem como os valores relativos, referentes ao total de exames analisados em relação aos parâmetros técnicos utilizados por cada uma das profissionais técnicas associada ao tipo de densidade mamográfica é apresentado na tabela 1.

Tabela 1:Resultado dos parâmetros técnicos adotados pelas Técnicas em Radiologia para os diferentes tipos de densidade mamária

\begin{tabular}{|c|c|c|c|c|c|c|c|}
\hline \multirow{3}{*}{$\begin{array}{l}\text { Densidade } \\
\text { da mama }\end{array}$} & \multirow{3}{*}{$\begin{array}{c}\text { Parâmetro } \\
\text { técnico }\end{array}$} & \multicolumn{6}{|c|}{ Técnica em Radiologia } \\
\hline & & \multicolumn{2}{|c|}{$\mathbf{A}$} & \multicolumn{2}{|c|}{$\mathbf{B}$} & \multicolumn{2}{|c|}{ C } \\
\hline & & 244 & $94 \%$ & 333 & $47 \%$ & 492 & $82 \%$ \\
\hline \multirow[t]{3}{*}{$\mathbf{I}$} & DOSE & 16 & $6 \%$ & 376 & $53 \%$ & 81 & $14 \%$ \\
\hline & CNT & 0 & $0 \%$ & 3 & $0 \%$ & 27 & $5 \%$ \\
\hline & STD & 238 & $93 \%$ & 229 & $57 \%$ & 370 & $88 \%$ \\
\hline \multirow[t]{3}{*}{ II } & DOSE & 13 & $5 \%$ & 159 & $39 \%$ & 44 & $10 \%$ \\
\hline & CNT & 5 & $2 \%$ & 16 & $4 \%$ & 6 & $1 \%$ \\
\hline & STD & 220 & $92 \%$ & 296 & $68 \%$ & 350 & $88 \%$ \\
\hline \multirow[t]{3}{*}{ III } & DOSE & 16 & $7 \%$ & 130 & $30 \%$ & 27 & $7 \%$ \\
\hline & CNT & 4 & $2 \%$ & 10 & $2 \%$ & 23 & $6 \%$ \\
\hline & STD & 168 & $90 \%$ & 352 & $81 \%$ & 373 & $91 \%$ \\
\hline \multirow[t]{2}{*}{ IV } & DOSE & 19 & $10 \%$ & 57 & $13 \%$ & 20 & $5 \%$ \\
\hline & CNT & 0 & $0 \%$ & 24 & $6 \%$ & 19 & $5 \%$ \\
\hline
\end{tabular}

Com base nos resultados apresentados observa-se que a profissional B utiliza melhor a tecnologia disponibilizada pelo equipamento, diversificando, ao máximo, o emprego das técnicas radiológicas para os diferentes tipos de densidade mamária. Observa-se, também, que esta profissional foi quem teve maior aproveitamento dos exames válidos, obtendo um total de 496 
exames (42\%), embora seus plantões sejam realizados no meio da semana, período em que geralmente é maior o fluxo de pacientes.

Em relação a profissional A, observa-se é que, mesmo trabalhando uma única vez na semana na mamografia, o que lhe vale $20 \%$ dos exames analisados, ela pouco varia os padrões da técnica radiográfica disponibilizados no equipamento. Isto mostra a falta de experiência da profissional em relação ao tipo de exame, além da insegurança observada em alguns momentos durante a utilização do equipamento para o emprego de uma técnica adequada para uma melhor qualidade de imagem.

Já em relação a profissional C foi observado o descompromisso em relação ao aprendizado de novos procedimentos técnicos, que possibilitariam melhora na qualidade da imagem. Também foi observado que a mesma variava suas técnicas apenas em casos considerados extremos pelo corpo clínico.

A não variação do parâmetro técnico na maioria dos exames radiográficos de ambas as profissionais não prejudicou o laudo final. Entretanto, observa-se que a qualidade da imagem obtida poderia ser melhor, bem como os valores de dose aplicados poderiam ser menores em alguns casos. O problema maior do sistema digital observado era a correção que as mesmas fizeram, através das ferramentas de sistema de imagem, para ajustar a qualidade da impressão final.

\section{CONCLUSÃO}

Como conclusão deste trabalho observa-se que, a falta de conhecimento e preparo no uso das diversas ferramentas tecnológicas, que são desenvolvidas e disponibilizadas aos trabalhadores, é uma das causas da não melhora nos processos de qualidade de imagem. Além disso, observa-se a necessidade de um processo de atualização continuada dos profissionais, principalmente na eficiência para redução das doses de exposição atendendo a política de saúde nacional e a legislação vigente. 


\section{AGRADECIMENTO}

Agradecemos à direção do hospital e a gerência do serviço por proporcionar à possibilidade de realização deste trabalho que servirá de base para a construção de um projeto maior de análise dos parâmetros técnicos empregados, da qualidade técnica dos profissionais, da qualidade dos serviços prestados a população, além das relações de dose empregadas em procedimentos de rastreamento. Agradecemos, também, aos profissionais médicos que, de alguma forma, contribuíram com informações e dicas para classificação das imagens radiográficas.

\section{REFERENCIAS}

1. BRASIL. Lei $\mathbf{n}^{0}$ 11.664. Dispõe sobre a efetivação de ações de saúde que assegurem a prevenção, a detecção, o tratamento e o seguimento dos cânceres do colo uterino e de mama, no âmbito do Sistema Único de Saúde - SUS. 28 abril 2008.

2. FOGAÇA, E.; GARROTE, L. Câncer de mama: atenção primária e detecção precoce. Arq Ciênc Saúde, v. 11, n. 3, p.179-181, 2004.

3. INCA. Programa Nacional de Controle do Câncer de Mama, Rio de Janeiro, Brasil, 2011. Disponível em: <http://www2.inca.gov.br/wps/wcm/connect/fad72d004eb684b68b379bf11 fae00ee/pncc_mama.pdf?MOD=AJPERES\&CACHEID=fad72d004eb684b68b379bf11fae00 ee>. Último acesso: 18 março 2014.

4. LEÃO, M.; PINTO, A.; BRAGA, D. Cuidados de Enfermagem nos Níveis de Prevenção da História Natural do Câncer de Mama. Percurso Acadêmico, v. 1, n. 2, p.264-280, 2011. Disponível em: <http://periodicos.pucminas.br/index.php/percursoacademico/article/view/ 2285/4149>. Último acesso: 13 março 2014.

5. LIMA-COSTA, M.; MATOS, D. Prevalência e fatores associados à realização da mamografia na faixa etária de 50-69 anos: um estudo baseado na Pesquisa Nacional por Amostra de Domicílios (2003). Cad. Saúde Pública, v. 23, n. 7, p.1665-1673, 2007. 
6. URBAN, L. et al. Recomendações do Colégio Brasileiro de Radiologia e Diagnóstico por Imagem, da Sociedade Brasileira de Mastologia e da Federação Brasileira das Associações de Ginecologia e Obstetrícia para rastreamento do câncer de mama por métodos de imagem. Radiol Bras, v. 45, n. 6, p.334-339, 2012. 